\title{
SARS-CoV-2 infection in Spanish children with chronic kidney pathologies
}

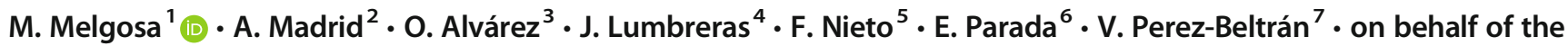 \\ Spanish Pediatric Nephrology Association
}

Received: 26 April 2020 / Revised: 26 April 2020 / Accepted: 1 May 2020 / Published online: 20 May 2020

(C) IPNA 2020

\begin{abstract}
Background There is little information about Coronavirus Disease 2019 (COVID-19) in children with underlying chronic renal pathologies.

Cases report From March until April 15, 2020, 16 children with chronic renal pathologies were diagnosed with COVID-19 in Spain. Of these, 6 had end-stage kidney disease (ESKD) ( 3 transplant recipients and 3 on chronic hemodialysis). The severity of symptoms was mild in all the patients, with little radiological involvement. Three patients were asymptomatic. Fever and upper respiratory symptoms were the most frequent findings. Basal glomerular filtration worsened in 3 patients; however, recovery was rapidly achieved with rehydration and drug dose adjustment. In 2 patients diagnosed with steroid-dependent nephrotic syndrome, COVID-19 provoked a disease relapse. None required oxygen therapy, and 7 could be managed as outpatients.

Conclusions COVID-19 disease appears to have a similar clinical course in children with underlying chronic renal pathologies, even in immunosuppressed cases, as in healthy children of the same age; however, special attention must be paid to fluid management and drug dose adjustment.
\end{abstract}

Keywords COVID-19 · CKD $\cdot$ Children · Nephrotic syndrome $\cdot$ Immunosuppression

\section{Introduction}

Coronavirus disease 2019 (COVID-19) is a novel viral disease caused by the Severe Acute Respiratory Syndrome Coronavirus 2 (SARS-CoV-2) virus. The original cases

M. Melgosa

marta.melgosa@salud.madrid.org
A. Madrid
amadrid@sjdhospitalbarcelona.org
O. Alvárez
olallaalvarezblanco@gmail.com
J. Lumbreras
jlumfer@gmail.com
F. Nieto
franciscoantonionieto@gmail.com
E. Parada
esterparada@yahoo.es
V. Perez-Beltrán
victorpbeltran@gmail.com

occurred in Wuhan, China, in December 2019 and rapidly spread to other areas worldwide, constituting a pandemic with unimaginable health and economic consequences.

Spain is currently one of the most affected countries, with more than 180,000 reported cases and more than 18,000

1 Pediatric Nephrology Department, La Paz University Hospital, Madrid, Spain

2 Pediatric Nephrology Department, Sant Joan de Deu University Hospital, Barcelona, Spain

3 Pediatric Nephrology Department, Gregorio Marañón University Hospital, Madrid, Spain

4 Pediatric Nephrology Department, Son Espases University Hospital, Palma de Mallorca, Spain

5 Pediatric Nephology Department, Malaga University Hospital, Málaga, Spain

6 Pediatric Nephrology Department, Joan XXIII University Hospital, Tarragona, Spain

Pediatric Nephrology Department, Vall d'Hebron University Hospital, Barcelona, Spain 
deaths; the first case was declared on January 31,2020 . At the present time, approximately 1000 patients aged younger than 19 years have been diagnosed; however, given that asymptomatic or mildly symptomatic children are not tested, the real number is probably much higher.

SARS-CoV-2 infection in children younger than 10 years of age has been described in approximately $1 \%$ of identified cases in China [1]. Although data on children is still limited, most series agree that cases are typically mild, even in infants [2,3].

Several reports regarding adults have reported advanced age and underlying pathologies as important risk factors for severe SARS-CoV-2 infection [3]; however, practically no information is available about the additional risk that chronic comorbidities pose to infected children.

The Spanish Pediatric Nephrology Association links all pediatric nephrologists working in Spain. The objective of this collaborative study is to describe the incidence and characteristics of SARS-CoV-2 infection in Spanish children with chronic renal pathology.

\section{Patients and methods}

A retrospective study was performed (March 1 to April 15, 2020) of children younger than 18 years infected with SARS$\mathrm{CoV}-2$ previously diagnosed with a chronic renal pathology. For inclusion in the study, SARS-CoV-2 polymerase chain reaction (PCR) result had to be positive. Both inpatients and outpatients were included. An online questionnaire was sent to all the members of the Spanish Pediatric Nephrology Association and was completed by nephrology pediatricians working at 43 hospitals.

Symptoms, analytical and radiological data, syndromic diagnosis, previous underlying disease, immunosuppressive treatment, treatment received for COVID-19 disease, and evolution data were collected.

\section{Results}

From March until April 15, 2020, 16 children (7 girls and 9 boys) with chronic renal pathologies were found by PCR to be positive for SARS-CoV-2. Of these, 6 were patients with ESKD: 3 had undergone renal transplant $(1.5,6$, and 10 years ago), and 3 were receiving chronic hemodialysis. Three of the 16 were children with chronic kidney disease (CKD) stages 23 , and 7 had a normal glomerular filtration rate (GFR). Clinical characteristics are detailed in Table 1.

A positive contact was present in half of the children. $62.5 \%$ of the children had respiratory symptoms (cough and/ or rhinorrhea), $50 \%$ had fever, and $25 \%$ had gastrointestinal symptoms. Three of the children were asymptomatic, and the test was performed because of their contact with a person who
Table 1 Demographic and clinical characteristics of patients $(n$ total $=$ 16)

\begin{tabular}{|c|c|}
\hline Patient characteristics & $N / n$ total \\
\hline $\operatorname{Sex}(\sigma: \varphi)$ & $(9: 7) / 16$ \\
\hline \multicolumn{2}{|l|}{ Age } \\
\hline Median age (range) & 12.32 years ( 1 month -17 years) \\
\hline$<1$ year old & $2 / 16$ \\
\hline $1-12$ years & $4 / 16$ \\
\hline$>12$ years & $10 / 16$ \\
\hline \multicolumn{2}{|l|}{ CKD stage } \\
\hline Normal GFR & $7 / 16$ \\
\hline CKD 2 & $2 / 16$ \\
\hline CKD 3 & $1 / 16$ \\
\hline \multicolumn{2}{|l|}{ ESKD } \\
\hline Hemodialysis & $3 / 16$ \\
\hline Peritoneal dialysis & $0 / 16$ \\
\hline Transplant & $3 / 16$ \\
\hline \multicolumn{2}{|l|}{ Underlying disease } \\
\hline Renal dysplasia & $5 / 16$ \\
\hline Nephrotic syndrome & $5 / 16$ \\
\hline Uropathy (megaureter/PUV) & $2 / 16$ \\
\hline IgA nephropathy & $1 / 16$ \\
\hline Scarring nephropathy & $1 / 16$ \\
\hline Vasculitis & $1 / 16$ \\
\hline Cortical necrosis & $1 / 16$ \\
\hline Exposure to contact & $8 / 16$ \\
\hline \multicolumn{2}{|l|}{ Signs and symptoms } \\
\hline Fever & $8 / 16$ \\
\hline Cough & $6 / 16$ \\
\hline Rhinorrhea & $6 / 16$ \\
\hline Gastrointestinal symptoms & $4 / 16$ \\
\hline Asymptomatic & $3 / 16$ \\
\hline Lymphopenia $(<1500$ cells/ $\mu \mathrm{l})$ & $4 / 12$ \\
\hline Abnormal X-ray & $6 / 10$ \\
\hline \multicolumn{2}{|l|}{ Respiratory support } \\
\hline None & $16 / 16$ \\
\hline Antiviral treatment & $6 / 16$ \\
\hline Lopinavir/ritonavir & $1 / 16$ \\
\hline Hydroxychloroquine & $6 / 16$ \\
\hline Others & $0 / 16$ \\
\hline Previous immunosuppression & $9 / 16$ \\
\hline Steroids & $7 / 16$ \\
\hline Cyclosporin A & $3 / 16$ \\
\hline Tacrolimus & $4 / 16$ \\
\hline Mycophenolate & $3 / 16$ \\
\hline Azathioprine & $1 / 16$ \\
\hline Everolimus & $1 / 16$ \\
\hline Levamisol & $1 / 16$ \\
\hline Hospitalization & $8 / 16$ \\
\hline Days of admission & $3 \pm 0.5$ days \\
\hline ICU admissions/deaths & $0 / \overline{0}$ \\
\hline Follow-up (median) & 19 days $(32-1)$ \\
\hline
\end{tabular}

GFR glomerular filtration rate, $C K D$ chronic kidney disease, ESKD endstage kidney disease, $P U V$ posterior urethral valve, $I C U$ intensive care unit

tested positive for SARS-CoV-2. Lymphopenia (defined as < 1500 cells $/ \mu$ l) was present in 4 of 12 patients for whom data were available. X-ray was performed in 10 patients, 6 of which were described as abnormal (4 diffuse infiltrates and 2 focal infiltrates). No patient needed oxygen therapy. 
Eight patients were hospitalized, and 8 were managed as outpatients. At admission, 3 patients ( 2 transplant recipients and 1 with CKD stage 2) were diagnosed with acute kidney injury (AKI) associated with a poor fluid intake; 1 patient had tacrolimus toxicity. All of them returned to basal GFR in a few days.

Of the 16 patients, 9 received chronic immunosuppression: 3 transplant recipients, 1 with vasculitis on chronic hemodialysis, 4 with nephrotic syndromes, and 1 with IgA nephropathy. In the vasculitis case, azathioprine was stopped; in the transplant recipients, mycophenolate was stopped (1 case) or reduced (1 case); and tacrolimus was reduced (1 case) or not modified (1 case). Everolimus was not modified.

Four infected patients were diagnosed with steroiddependent nephrotic syndrome: 2 were asymptomatic, and maintenance immunosuppression was not modified; in the other 2, coronavirus infection provoked a nephrotic relapse, but prednisone treatment could be administered without complications.

In 6 of the 16 cases, some antiviral treatment was prescribed: 5 patients received only hydroxychloroquine, and 1 first received lopinavir-ritonavir, which was interrupted by gastrointestinal secondary effects, and then hydroxychloroquine.

With a median follow-up of 19 (1-32) days from COVID19 diagnosis, clinical recovery has been completed in all the patients. No child required admission to the pediatric intensive care unit or died.

\section{Discussion}

Data regarding epidemiological or clinical features of children infected with SARS-CoV-2 are limited; however, it appears that the incidence is much lower than in adults. In the largest pediatric Chinese series published to date, approximately $2 \%$ of the 44,672 confirmed cases were children aged $0-19$ years [4]. In Italian data, only $1.2 \%$ of the cases reported were children [5]. In the USA, among 149,086 cases reported for which age was known, $1.7 \%$ were among children aged $<18$ years [6]. In Spain, in official data actualized to April 13, 2020 [7], only 535 of 113,368 confirmed cases were children $<15$ years $(0.47 \%)$. This lower incidence probably reflects that only symptomatic patients have been tested in Spain.

Although CKD appears to be associated with enhanced risk of severe COVID-19 disease in adults [8], little information is available about pediatric patients with CKD. In our study, 16 children with an underlying chronic renal pathology were diagnosed: 3 were asymptomatic, and test was indicated due to their renal pathology and contact with a person who tested positive for SARS-CoV-2. Using as reference the 2 Spanish Pediatric Chronic Kidney Registries that collect data from children with ESKD (dialysis or transplant) and from children with predialysis CKD (stages 2-5), we can estimate an incidence of $0.61 \%$ for children with ESKD and $0.35 \%$ for predialysis stages, similar to that of the general pediatric population.

The clinical presentation is typically mild [1]. In a recent USA report [6], fever, cough, and especially shortness of breath are commonly reported among adult patients $(71 \%$, $80 \%$, and $43 \%$, respectively) but are less frequently found in children $(56 \%, 54 \%$, and $13 \%$, respectively) [8]. In our series, 3 of 16 patients were asymptomatic, and fever and/or cough were present in only half of the patients. Remarkably, none of our patients required oxygen therapy. Lymphopenia was an unusual finding as previously reported in pediatric series [1].

In 2 patients with corticodependent nephrotic syndrome receiving maintenance treatment with immunosuppressors, SARS-CoV-2 infection provoked a relapse, but prednisone treatment response was good in both cases.

Acute kidney injury (AKI) is not commonly reported in COVID-19 disease [9], but when present, it is associated with a poorer prognosis [10]. Three children were diagnosed with AKI, with an increase in serum creatinine to 1.5 times baseline (stage $1 \mathrm{AKIN} / \mathrm{KDIGO}$ classification). In the 3 cases ( 2 transplant recipients and an adolescent with ERC stage 2), a prerenal component was present, and in 1 of them, tacrolimus toxicity was also identified. All 3 patients achieved basal GFR in a few days.

Nine of the 16 children received chronic immunosuppression. Although data for immunosuppressed children are scarce, surprisingly, they did not appear to evolve differently from the immunocompetent children. A study performed by the European Society for Gastroenterology [11] found children with inflammatory bowel disease who presented COVID-19 disease with a mild outpatient course. In a multicentric study of children receiving anticancer chemotherapy, most of the cases have a mild or asymptomatic clinical course [12]. A recent study performed in Madrid (unpublished work) collecting data from 8 immunocompromised children with COVID-19 had found similar results.

Special consideration must be given to transplant recipients. Data from Hospital Papa Giovanni XXIII of Bergamo [13] shows that, among approximately 200 pediatric liver transplant recipients controlled in this center, none had developed clinical pulmonary disease, despite 3 having tested positive for SARS-CoV-2. In our own experience, among approximately 420 children with a functioning kidney transplant controlled in pediatric nephrology departments in Spain, only 3 patients have been diagnosed with COVID-19.

There are no general rules about immunosuppression management. Based on experience with other viruses in the transplantation field, antimetabolites are typically reduced or discontinued, but calcineurin inhibitors are maintained if the clinical course is not severe. Evolutions have been favorable, even in cases with any modification that has been done. 
Antiviral treatment was prescribed according to general practice advice for the Spanish pediatric population [14]. One patient initially received lopinavir/ritonavir, but administration had to be stopped due to digestive intolerance; it was replaced with hydroxychloroquine. The other 8 patients were only treated with hydroxychloroquine. We must be careful when recommending a specific antiviral treatment for this group of patients, given that pharmacological interactions are very frequent. It is also important to remember that doses must to be adjusted to CKD degree.

In conclusion, in our experience, children with chronic renal pathologies, even with immunosuppression, and COVID19 disease show the same evolution as healthy children of the same age. Clinical course is typically mild. Renal acute failure could appear if anorexia limits fluid intake. Drug interaction must be kept in mind. Hospitalization should be considered only when the clinical situation suggests it, and these patients can be managed as outpatients if close monitoring is possible.

Data availability All data generated or analyzed during this study are included in this published article.

\section{Compliance with ethical standards}

Conflict of interest The authors declare that they have no conflict of interest.

Ethics approval Not applicable.

Consent to participate Not applicable.

Consent for publication Not applicable.

\section{References}

1. Lu X, Zhang L, Du H et al (2020) Chinese Pediatric Novel Coronavirus Study Team (2020). SARS-CoV-2 infection in children. N Engl J Med. https://doi.org/10.1056/NEJMc2005073 [Epub ahead of print]

2. Dong Y, Mo X, Hu Y et al (2020) Epidemiological characteristics of 2143 pediatric patients with 2019 coronavirus disease in China. Pediatrics. https://doi.org/10.1542/peds.2020-0702
3. Ludvigsson JF (2020) Systematic review of Covid-19 in children shows milder cases and better prognosis than adults. Acta Pediatrica. https://doi.org/10.1111/apa.15270 [Epub ahead of print]

4. World health Organization. Report of the WHO-China Joint Mission on Coronavirus Disease 2019 (COVID-19) [Internet]. 2020. https://www.who.int/docs/default-source/coronaviruse/whochina-joint-mission-on-covid-19-final-report.pdf

5. Livingston E, Bucher K (2020) Coronavirus disease 2019 (COVID19) in Italy. JAMA. https://doi.org/10.1001/jama.2020.4344 [Epub ahead of print]

6. Coronavirus Disease 2019 in Children - United States, February 12-April 2, 2020.CDC COVID-19 Response Team (2020).MMWR Morb Mortal Wkly Rep. 10;69 (14):422-426

7. Red Nacional de Vigilancia Epidemiológica. Situación de COVID19 en España. Informe 22 [Internet]. $2020 \mathrm{https}: / / \mathrm{www}$. isciii.es/ QueHacemos/Servicios/VigilanciaSaludPublicaRENAVE/ EnfermedadesTransmisibles/Documents/INFORMES/Informes\% 20COVID-19/Informe $\% 20 \mathrm{n} \% \mathrm{C} 2 \% \mathrm{BA} \% 2022 . \% 20$ Situaci\%C3\% B3n\%20de\%20COVID-19\%20en\%20Espa\%C3\%B1a\%20a\% 2013\%20de\%20abril\%20de\%202020.pdf. Acces Apr 18

8. Henry BM, Lippi G (2020) Chronic kidney disease is associated with severe coronavirus disease 2019 (COVID-19) infection. Int Urol Nephrol. https://doi.org/10.1007/s11255-020-02451-9 [Epub ahead of print]

9. Wang L, Li X, Chen $\mathrm{H}$ et al (2020) Coronavirus disease 19 infection does not result in acute kidney injury: an analysis of 116 hospitalized patients from Wuhan. China Am J Nephrol 31:1-6

10. Cheng Y, Luo R, Wang K et al (2020) Kidney impairment is associated with in-hospital death of COVID-19 patients. Kidney Int. https://doi.org/10.1016/j.kint.2020.03.005 [Epub ahead of print]

11. Turner D, Huang Y, Martín-de-Carpi J et al (2020) COVID-19 and paediatric inflammatory bowel diseases: global experience and provisional guidance (march 2020) from the Paediatric IBD Porto group of ESPGHAN. J Pediatr Gastroenterol Nutr. https://doi.org/ 10.1097/MPG.0000000000002729 [Epub ahead of print]

12. Hrusak O, Kalina T, Wolf $J$ et al (2020) Flash survey on severe acute respiratory syndrome coronavirus- 2 infections in paediatric patients on anticancer treatment. Eur J Cancer 132:11-16 [Epub ahead of print]

13. D'Antiga L (2020) Coronaviruses and immunosuppressed patients. The facts during the third epidemic. Liver Transpl. https://doi.org/ 10.1002/lt.25756 [Epub ahead of print]

14. Calvo C, García López-Hortelano M, de Carlos Vicente JC, Vázquez Martínez JL, Grupo de trabajo de la Asociación Española de Pediatría para el brote de infección por Coronavirus (2020) An Pediatr. https://doi.org/10.1016/j.anpedi.2020.02.001 [Epub ahead of print]

Publisher's note Springer Nature remains neutral with regard to jurisdictional claims in published maps and institutional affiliations. 